\title{
Nanofabrication of SOI-Based Photonic Waveguide Resonators for Gravimetric Molecule Detection ${ }^{+}$
}

\author{
Tony Granz 1,2,*, Julia Sophie Böke 1,3, Gerry Hamdana 1,2, Michael Martens 2,3, Arijit Misra 1, \\ Stefan Preußler ${ }^{1}$, Meinhard Schilling 2,3, Erwin Peiner ${ }^{1,2}$, Thomas Schneider ${ }^{1,2}$, Andreas Waag 1,2 \\ and Hutomo Suryo Wasisto ${ }^{1,2, *}$ \\ 1 Institut für Halbleitertechnik (IHT), Technische Universität Braunschweig, 38106 Braunschweig, Germany; \\ j.boeke@tu-braunschweig.de (J.S.B.); g.hamdana@tu-braunschweig.de (G.H.); \\ arijit.misra@ihf.tu-bs.de (A.M.); stefan.preussler@ihf.tu-bs.de (S.P.); e.peiner@tu-bs.de (E.P.); \\ thomas.schneider@ihf.tu-bs.de (T.S.); a.waag@tu-bs.de (A.W.) \\ 2 Laboratory for Emerging Nanometrology (LENA), Technische Universität Braunschweig, \\ 38106 Braunschweig, Germany; m.martens@tu-bs.de (M.M.); m.schilling@tu-bs.de (M.S.) \\ 3 Institut für Elektrische Messtechnik und Grundlagen der Elektrotechnik (EMG), Technische Universität \\ Braunschweig, 38106 Braunschweig, Germany \\ * Correspondence: t.granz@tu-braunschweig.de (T.G.); h.wasisto@tu-braunschweig.de (H.S.W.) \\ † Presented at the Eurosensors 2018 Conference, Graz, Austria, 9-12 September 2018. \\ Published: 13 February 2019
}

\begin{abstract}
A silicon photonic microresonator comprising two curved vertical grating couplers and a single suspended Si nanowaveguide (NWG) is developed to investigate the giant enhanced Brillouin scattering in subwavelength NWGs caused by photon-phonon interaction. Finite element modelling based on COMSOL Multiphysics is conducted to optimize the critical device parameters (e.g., waveguide width, height, and length). As the smallest structures that need to be resolved are down to $\sim 15 \mathrm{~nm}$ in size, electron-beam nanolithography is employed. In this case, dosage tests are carried out to minimize proximity charging effects during the nanopatterning of the silicon-oninsulator (SOI) surface, resulting in appropriate adaptive current area dosage distributions for the periodic gratings, couplers peripheral areas, and NWG, respectively. Furthermore, an enhanced inductively coupled plasma dry reactive ion etching (ICP-DRIE) process at a cryogenic temperature is used to realize smooth vertical sidewalls. Finally, buffered hydrofluoric acid (BHF)-based wet chemical etching is carried out to remove the buried oxide resulting in a suspended Si waveguide.
\end{abstract}

Keywords: nanowaveguide; silicon photonics; optical communication; integrated optics; silicon technology

\section{Introduction}

Integrated silicon photonics is a fast growing technology, which is especially demanded in longhaul communication and data centers. This results in a rapid technology advancement and opens new markets and applications in metrology, high speed computing, and biochemical sensing. The driving force behind the industrial and academic interest is the ability to use CMOS-like fabrication standards for high-volume production at low cost [1]. Therefore, comprehensive effort has been made to provide silicon photonic devices (e.g., optical sources, optical modulators, photodetectors, and integrated packages for all-optical Si based photonic circuits). However, there are only a few approaches in literature to implement silicon photonic devices as biochemical detection platforms [1]. For a monolithic integration of biochemical sensing nanodevices on a single photonic chip, a suitable gravimetrical molecule detection platform has to be investigated to obtain a compact optomechanical sensing systems. 
Differentiating from the other micro-/nanoelectromechanical resonant devices that are typically based on piezoresistive or piezoelectric transduction sensing mechanisms (e.g., silicon cantilevers [24] and vertical nanowire resonators [5,6]), this work shows an approach to design and fabricate microresonators based on the giant enhanced photon-phonon interaction, so-called Brillouin effect, which occurs in sub-wavelength waveguides [7].

\section{Simulations}

COMSOL Multiphysics ${ }^{\circledR}$ simulations have been carried out to define critical device parameters and estimate the influence of manufacturing tolerances. A waveguide width of $210 \mathrm{~nm}$ has shown the most optimized results providing agreement between optical confinement of the guided mode inside the core and a strong $x$-component of TE-mode at the interface between silicon and air (Figure 1b) [7]. In this case, a slight NWG width variation of $\pm 15 \mathrm{~nm}$ may result in a significant decrease of field intensity to almost zero for width sizes of $195 \mathrm{~nm}$ and $240 \mathrm{~nm}$ (Figure 1a,d) and 50\% for $225 \mathrm{~nm}$ (Figure 1c). Moreover, optical forces were also calculated, which are divided into three main contributors (i.e., electrostrictive body force, boundary induced electrostriction, and radiation pressure). Calculation was done by exporting simulated TE-mode field components into MATLAB, determining force components in accordance to [8] and re-importing them to COMSOL. The simulations show that imperfection of only a few nanometers from the optimal NWG geometry will significantly influence the resonator performances. Therefore, a highly precise nanofabrication process has become a prerequisite to fulfill the device specification. Furthermore, optical and acoustical confinements of the associate waves have to be guaranteed by exposing the NWG from every side.
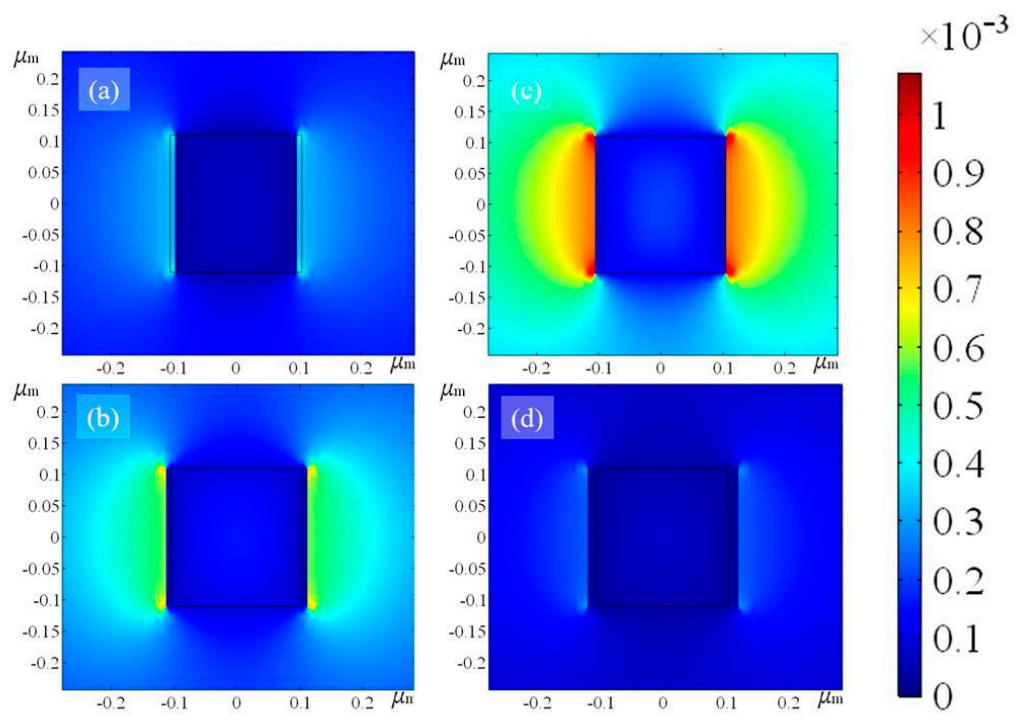

Figure 1. COMSOL eigenmode analysis showing the normalized electric field $x$-component of TEmode for waveguide widths of (a) $195 \mathrm{~nm}$, (b) $210 \mathrm{~nm}$, (c) $225 \mathrm{~nm}$, and (d) $240 \mathrm{~nm}$ at a constant waveguide height of $220 \mathrm{~nm}$.

\section{Nanofabrication}

The high-precision nanofabrication process consists of five sequential manufacturing steps. At the beginning of device fabrication, a careful basic cleaning process was performed by boiling the sample at $80^{\circ} \mathrm{C}$ in $\mathrm{H}_{2} \mathrm{SO}_{4}+\mathrm{H}_{2} \mathrm{O}_{2}$ (1:1) and dipping it into $\mathrm{HF}$ acid to remove the natural oxide layer. To accomplish the necessary accuracy, electron-beam lithography tests were performed by a Zeiss SUPRA 35 FE-SEM with Raith ElphyPlus add-on using an acceleration voltage $30 \mathrm{kV}$ and a minimum pixel size of $16 \mathrm{~nm}$. Therefore, the SOI chip was coated with an $80 \mathrm{~nm}$ special e-beam lithography resist AR-P 6200.04 (Figure 2a) for lift-off process. An additional $30 \mathrm{~nm}$ protective coating of AR-PC 
5090 defines a thin conductive layer on top of the e-beam resist, which avoids e-beam deflection caused by charging of the buried oxide (BOX) underneath the thin Si device layer $(220 \mathrm{~nm})$. By designing a $1000 \times 1000 \mu \mathrm{m}^{2}$ dosage test matrix ranging from $55-110 \mu \mathrm{C} / \mathrm{cm}^{2}$, optimal area dosages for the periodic gratings and the waveguide were identified to avoid proximity effects. After e-beam exposure, a thin chromium-etching mask film was deposited by e-beam evaporation, in which a subsequently lift-off process was performed by using AR 600-71 remover and ultrasonic bath (Figure $2 b)$. An ICP-DRIE recipe using $\mathrm{SF}_{6} / \mathrm{O}_{2}$ plasma was chosen and optimized to obtain a highly controlled etching rate of $60 \mathrm{~nm} / \mathrm{min}$ and orthogonal sidewalls (Figure 2c). To guarantee a homogenous thickness of grating coupler and nanowaveguide across the whole device, the etching process can be automatically stopped at the BOX layer. Afterwards, a photolithography step involving AZ $5215 \mathrm{E}$ positive resist was used to expose the NWG surroundings for the subsequent wet chemical etching (Figure 2d). Finally, in the last step, the suspension of the NWG was obtained by removing the $3 \mu \mathrm{m}$ BOX-layer underneath the NWG (Figure 2d) using hydrofluoric acid (HF) wet chemical etching step for $4 \mathrm{~min}$, which leads to a total optical and acoustical isolation between the NWG and the substrate, leading to a wave confinement. The whole process sequence is shown in Figure 2.
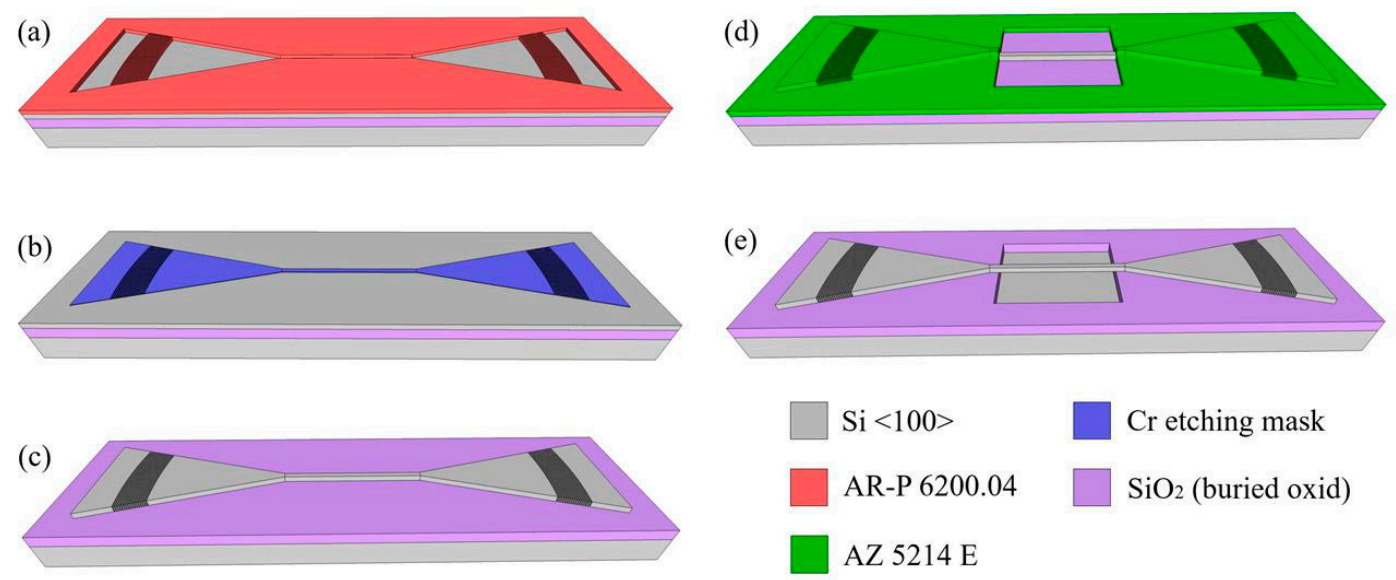

Figure 2. Nanofabrication process sequence on thin-film SOI wafer-device layer (220 $\mathrm{nm})$, buried oxide $(3 \mu \mathrm{m})$, handle layer $(675 \mu \mathrm{m})$ : (a) e-beam lithography using $80 \mathrm{~nm}$ AR-P 6200.04 (CSAR 62) resist; (b) chromium deposition via e-beam evaporation and subsequent lift-off process; (c) ICP-DRIE of device layer by $\mathrm{SF}_{6} / \mathrm{O}_{2}$ plasma; (d) combined photolithography using positive resist $\mathrm{AZ} 5214 \mathrm{E}$; (e) suspension of NWG by HF-based wet etching.

\section{Results and Discussion}

Figure 3a-e shows the critical areas of the opto-mechanical micro resonator. The exact compliances of the grating couplers specification are crucial to cut off all diffractive orders in the cladding, so that only orders in the waveguide layer exists. According to simulation results, a device with grating width of $330 \mathrm{~nm}$ and interstices of $300 \mathrm{~nm}$ can obtain the optimum guided mode coupling. Figure $3 \mathrm{~b}$ shows the bended periodic silicon lines of the grating coupler after dosage test optimization and ICP-DRIE process. Here, e-beam area dosages of $70 \mu \mathrm{C} / \mathrm{cm}^{2}$ and $100 \mu \mathrm{C} / \mathrm{cm}^{2}$ were applied for structuring the gratings and the NWG, respectively. In the current device status, the necessary dimension could be realized. However, ripples and sidewall imperfections are still visible, which will affect the resonators performance negatively. Figure $3 c$ shows that the ripples are transferred from the chromium etching mask. Hence, an additional tuning between the beam properties and the resist in this region is mandatory. A smaller pixel size will smoothen the edges of the gratings and the NWG itself. Additionally, a reduction of the oxygen content in the ICP-DRIE can reduce the sidewall passivation during the dry etching process and consequently decrease the sidewall angle of $\sim 20^{\circ}$ for stepper etching flanks. Alternatively, a higher ICP-power is also conceivable as an alternative recipe adjustment, although higher etching rate and plasma damages will follow as its consequence and therefore have to be considered in this case. 
Regardless of the required optimization of the device processing steps, the critical dimensions of the opto-mechanical resonators (i.e., $210 \mathrm{~nm}$ ) could already be obtained during area dosage test and e-beam resist optimization (Figure 3d). Furthermore, a transfer area of the solidly mounted NWG to suspended NWG has been realized with a continuous suspended NWG length of $200 \mu \mathrm{m}$. The high aspect ratio of 900 leads to a slightly bending of the waveguide (Figure 3a), which could be avoided with buried perpendicular oxide bridges to provide mechanical support along the NWG. Besides SEM analysis, a forward SBS measurement setup will be used to evaluate the resonator manufacturing quality by power-conversion and quality-factor measurements.
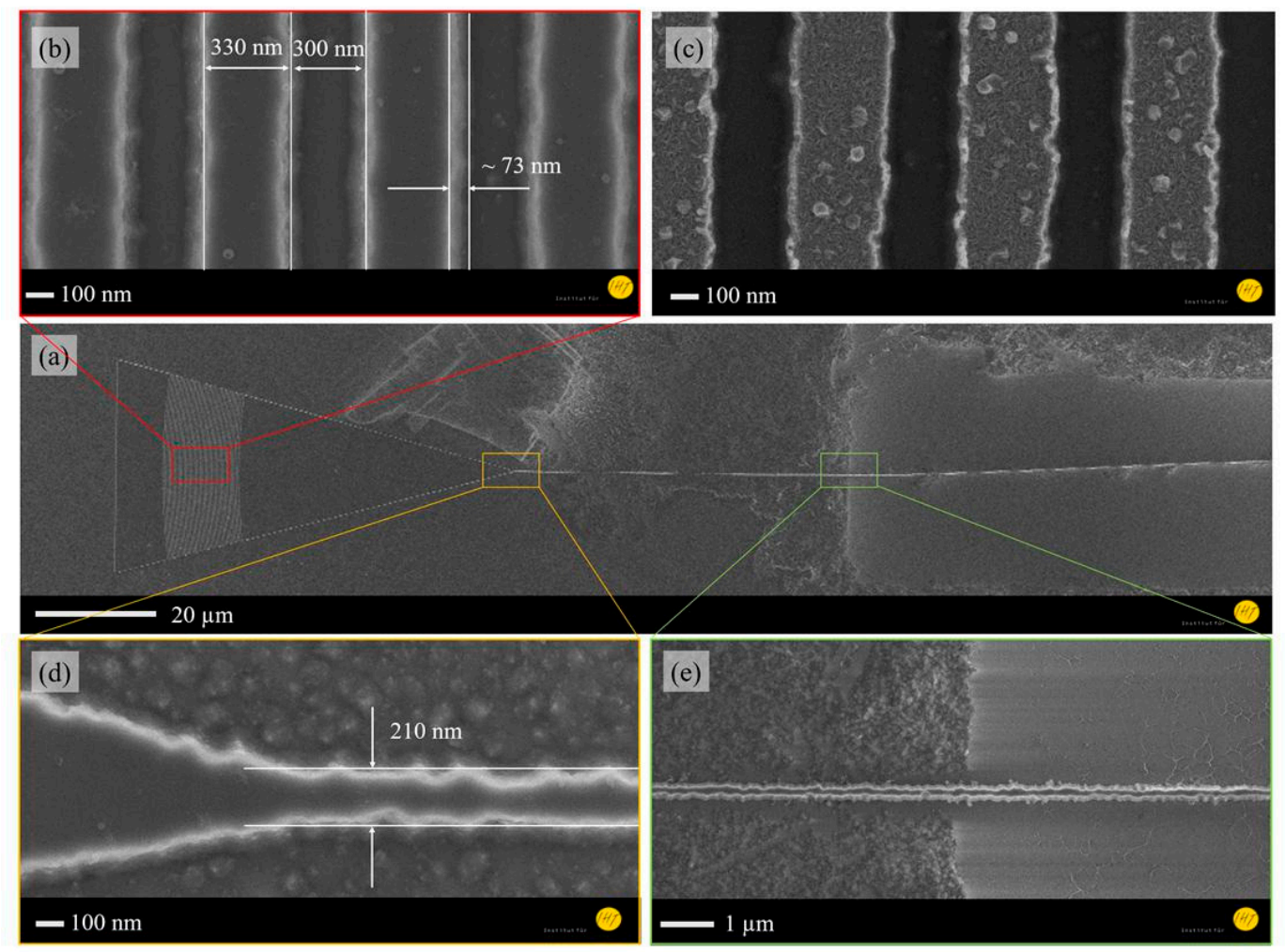

Figure 3. SEM images depicting (a) left part of microresonator comprising a grating coupler with connected NWG and suspended region, (b) a grating with line width of $330 \mathrm{~nm}$ and gap of $300 \mathrm{~nm}$, (c) Cr mask used for ICP-DRIE, (d) interface between grating coupler und NWG, and (e) transfer area of solidly mounted NWG to suspended NWG.

Author Contributions: T.G., J.S.B., M.M., and H.S.W. conceived and designed the experiments; T.G. and J.S.B. performed the experiments; T.G., J.S.B., M.M., G.H., S.P., A.M., E.P. and H.S.W. analyzed the data; M.S., T.S, A.W., and H.S.W. contributed reagents/materials/analysis tools; T.G. wrote the paper. H.S.W. revised the paper; H.S.W. and A.W. lead the project funded by N-MWK.

Acknowledgments: The authors would like to thank A. Schmidt, J. Breitfelder, and M. Rühmann for their valuable technical assistance. This work is performed within project of LENA-OptoSense funded by the Lower Saxony Ministry for Science and Culture (N-MWK), Germany.

Conflicts of Interest: The authors declare no conflict of interest.

\section{References}

1. Lim, A.E.-J.; Song, J.; Fang, Q.; Li, C.; Tu, X.; Duan, N.; Chen, K.K.; Tern, R.P.-C.; Liow, T.-Y. Review of silicon photonics foundry efforts. IEEE J. Sel. Top. Quantum Electron. 2014, 20, 8300112.

2. Wasisto, H.S.; Merzsch, S.; Uhde, E.; Waag, A.; Peiner E. Handheld personal airborne nanoparticle detector based on microelectromechanical silicon resonant cantilever. Microelectron. Eng. 2015, 145, 96-103. 
3. Wasisto, H.S.; Merzsch, S.; Waag, A.; Uhde, E.; Salthammer, T.; Peiner, E. Airborne engineered nanoparticle mass sensor based on a silicon resonant cantilever. Sens. Actuator B 2013, 180, 77-89.

4. Wasisto, H.S.; Merzsch, S.; Steib, F.; Waag, A.; Peiner E. Vertical silicon nanowire array-patterned microcantilever resonators for enhanced detection of cigarette smoke aerosols. Micro Nano Lett. 2014, 9, 676-679.

5. Wasisto, H.S.; Merzsch, S.; Stranz, A.; Waag, A.; Uhde, E.; Salthammer, T.; Peiner, E. Femtogram aerosol nanoparticle mass sensing utilising vertical silicon nanowire resonators. Micro Nano Lett. 2013, 8, 554-558.

6. Wasisto, H.S.; Merzsch, S.; Stranz, A.; Waag, A.; Uhde, E.; Salthammer, T.; Peiner, E. Silicon nanowire resonators: Aerosol nanoparticle mass sensing in the workplace. IEEE Nanotechnol. Mag. 2013, 7, 18-23.

7. Rakich, P.; Reinke, C.; Camacho, R.; Davids, P.; Wang, Z. Giant enhancement of stimulated Brillouin scattering in the subwavelength limit. Phys. Rev. 2012, X2, 011008.

8. Qiu, W.; Rakich, P.; Soljacic, M.; Wang, Z. Stimulated Brillouin Scattering in Nanoscale Silicon Step-index Waveguides: A General Framework of Selection Rules and Calculating SBS Gain. Opt. Express 2013, 21, 31402-31419.

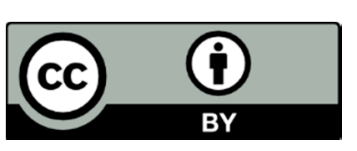

(C) 2019 by the authors. Licensee MDPI, Basel, Switzerland. This article is an open access article distributed under the terms and conditions of the Creative Commons Attribution (CC BY) license (http://creativecommons.org/licenses/by/4.0/). 